\title{
Sponsor Study Reference Identifier
}

National Cancer Institute

\section{Source}

National Cancer Institute. Sponsor Study Reference Identifier. NCI Thesaurus. Code C135009.

The reference identifier that is assigned by the sponsor and by which the study is known to the sponsor. 